\title{
Assessment of Community Led Total Sanitation Achievements and Its Associated Factors in Rural Kebeles of Adama Woreda, East Shoa Zone, Oromia, Ethiopia
}

\author{
Teshome Ergena Tuli ${ }^{1, *}$, Asfaw Ergena Tuli², Worku Dugasa Girsha ${ }^{3}$ \\ ${ }^{1}$ Department of Public Health, Adama General Hospital and Medical College, Adama, Ethiopia \\ ${ }^{2}$ Department of Agricultural Service and Value Chain, Ambo University, Ambo, Ethiopia \\ ${ }^{3}$ Department of Public Health, Adama Hospital Medical College, Adama, Ethiopia \\ Email address: \\ teshomeergena34@gmail.com (T. E. Tuli),ergenase@yahoo.com (A. E. Tuli), dugassaworku@gmail.com (W. D. Girsha) \\ ${ }^{*}$ Corresponding author
}

\section{To cite this article:}

Teshome Ergena Tuli, Asfaw Ergena Tuli, Worku Dugasa Girsha. Assessment of Community Led Total Sanitation Achievements and Its Associated Factors in Rural Kebeles of Adama Woreda, East Shoa Zone, Oromia, Ethiopia. Science Journal of Public Health .

Vol. 5, No. 4, 2017, pp. 313-320. doi: 10.11648/j.sjph.20170504.16

Received: April 2, 2017; Accepted: April 15, 2017; Published: June 26, 2017

\begin{abstract}
Ethiopia has made important improvement concerning entire water, sanitation and hygiene access in the past era on the other hand still significant problems present which varies from rural to urban. Due to limited access to safe water, inadequate sanitation and hygiene services, $60-80 \%$ preventable communicable diseases attributed in the country.For controlling, community led total sanitation program introduced in 2013. This is aimed to assess community led total sanitation achievements and its associated factors in rural kebeles. A cross-sectional study with cluster, probability proportion and simple random sampling techniques employed to collect data from 604 households. Data were collected through interview and observational checklist. The study revealed that, only 51(8.6\%) respondents answered community led total sanitation achieved. Sex $(A O R=4.72,95 \% \mathrm{CI}=1.09-8.48, \mathrm{p}=0.01$ ), monthly income (AOR $=2.00,95 \% \mathrm{CI}=1.09-3.69, \mathrm{p}=0.03$ ), open dump in the yard $(\mathrm{AOR}=2.03,95 \% \mathrm{CI}=1.11-3.72, \mathrm{p}=0.005)$ and triggering step $(\mathrm{AOR}=3.54,95 \% \mathrm{CI}=1.41-8.89, \mathrm{p}=0.003)$ significantly allied to community led total sanitation achievement. This finding concluded that, community led total sanitation achievement in the study areas were very lower than the areas posted open defecation free. Health workers, community representatives and local authorities must give special emphasis to improve the coverage.
\end{abstract}

Keywords: Community Led Total Sanitation Achievement, Latrine Utilization, Hand Washing Practices, Access Safe Water Supply

\section{Introduction}

According to WHO/UNICEF-joint monitoring program updated in 2012, sanitation is commonfor many international organizations to use access to hygienic sanitation facilities as a measure of progress in the fight against poverty, disease, and death [1]. Globally, still one in three (2.4 billion) people without improved sanitation, including one in eight (946 million) people practicing open defecation. Regarding the rural parts, still seven in ten people lack improved sanitation facilities and nine out of ten $(90 \%)$ people practicing open defecation [2].
Healthy epidemiological studies by Esrey displayed, sanitation is one of the biggest development challenges in most of developing countries. Pit latrines, when properly used can reduce diarrhea by $36 \%$ or more, cholera by $66 \%$, and worm infestations by between 12 to $86 \%$ [3]. A crosssectional study conducted in 2013 on the impact of e Indian total sanitation campaign on latrine coverage and use showed that over a third $(37 \%)$ of the members of households with latrines never defecating in their latrines [4] and also the same study method in rural India at 2015 resulted, improved conditions of sanitation and hygiene practices are associated with reducing prevalence of stunting [5].

Community led total sanitation provides techniques of 
unites the community to commit to using sanitary latrines and hygienic behavior, and understands that the process is a shift towards a zero subsidy approach rather than providing them with money to construct latrine [6]. In Africa, community led total sanitation has various benefits in comparison with the traditional subsidy led approach in case ofidentifying natural leaders who support the design and construction of subsidy free latrines and providing awareness for community about methods of disease transmission due to lack of proper sanitation and means of reduction [7]. In subSaharan Africa, majority of the countries not achieved open defecation free due to country's economicand only $30 \%$ of the rural population of sub-Saharan African countries was using improved sanitation facilities by $2015[8,9]$.

Ethiopia like many other countries in Sub-Saharan Africa has low levels of WASH facilities and practices. Only $4 \%$ of households use improved toilet facilities that are not shared with other households (14\% in urban and $2 \%$ rural areas) whereas the rests (57\% in rural and $43 \%$ urban areas) use non-improved toilet facility that means an open pit latrine or pit latrine without slabs and only $29 \%$ (28.3 million) declined open defecation $[2,10]$.

A study conducted in Southern nation nationality and people region of Ethiopia revealed that the number of household latrine coverage, in few years, grew from $16 \%$ to $94 \%$ in Mirab Abaya and $10 \%$ to $69 \%$ in Alaba [11] as well as open defecation free villages are also being promoted to improve the utilization of latrines in the region [12]. $41.6 \%$ of the households living in rural of Bahir Dar Zuria district lacked pit latrines [13] and $86.8 \%$ of households use latrine in Dembiaworeda, according to study conducted in 2013 [14].

Household's income is associated to availability of improved of sanitation facilities, which were households that had a higher income, have improved of sanitation facilities than lower incomes according to finding result in rural communities of LemoWoreda, Hadiya Zone, Southern Ethiopia in 2014 [15]. Provision of adequate sanitation facilities is not only a socioeconomic and developmental issue, but also an issue of self-respect, human dignity and public health [16]. A large proportion of Abomsa town residents have a latrine (89\%), only $36 \%$ households have an improved facility (e.g. with slab) and a substantial minority of households $(10 \%)$ still rely upon open defecation. In rural areas, fewer households have latrines (41\%) but far fewer are improved $(2 \%)$ and the majority practice open defecation [17].

Regardingwater supply, it is associated with a $15 \%$ reduction of diarrhea and a combined safe water supply, sanitation and hygiene can reduce diarrhea by 65 [3]. In Ethiopia, only $16 \%$ (piped and a protected well) and $12 \%$ of a protected spring were in rural households [18] and 53\% households travel 30 minutes or more to fetch their drinking water (19\% in urban and $62 \%$ rural areas) [19]. The study on access to safe drinking water and availability of environmental sanitation facilities among Dukem town households in 2013 pointed that almost all the households had access to improved water supply and quite large of them are unimproved toilet facility [20]. and another an assessment of urban water supply and sanitation in Ambo town, Oromia Region on March 2011, indicated that water and sanitation inaccessibility has a great deal to health, socioeconomic and environmental impacts [21].

Hand hygiene is the most simplest and effective measure to prevent infections and account $50 \%$ of health care associated infection due to hand of health care providers [18]. Studies on hand washing frequency, motivators and barriers have primarily been performed in stable developing country contexts. Curtis et al. performed an eleven country study and found in structured observations that $17 \%$ of care givers washed hands with soap after defecation, while $45 \%$ rinsed hands with water alone [22]. Hand washing with soap is more effective at hand decontamination than washing with water alone [23]. After contact with stools hand washing with soap and water can reduce diarrheal disease by $35 \%$ [3]. A study in three refugee camps by Biran et al. (in Kenya, Ethiopia and Thailand) found that soap was used for hand washing $20 \%$ after of toilet use events due to lack of free soap and preference to use soap for washing [24].

The study conducted in Denbia district among people living in rural areas in 2013 pointed hand washing practice after latrinewith access to all essential supplies were very low $(26.9 \%)$ and the presence of $\leq 5$ years children, job of mother farmer and rare cleaning of latrine were factors negatively associated as well as the educational status of mothers, presence of secondary school student, the presence of the door, frequency of latrine construction and hygienic condition of latrine were positively associated with latrine utilization [14]. Another study in kersaworeda by 2010 showed $8.3 \%$ respondent's practices hand washing after visited latrine [25].

Community led total sanitation program introduced to Ethiopia in 2006 which used as an entry point strategy [26]. Accordingly Adama district health report updated 2016, community led total sanitation achieved in ten rural kebeles and posted end open defecation [27].

The identifying of status of community led total sanitation achievement and its associated factors in the study areas will facilitate better understanding of the problems and influence organizations and decision making at all levels from communities to national levels. Therefore, this study was assessed community led total sanitation achievement and identified its associated factors in rural kebeles of Adama district.

\section{Methods and Materials}

\subsection{Study Design and Setting}

A community based cross-sectional study design with both descriptive and analytical components was used to assess community led total sanitation achievement and its' associated factors. The study was carried out in five rural kebeles of Adama district which far $99 \mathrm{~km}$ in the eastern 
of Addis Ababa, Ethiopia. The study population was all households in the selected ruralkebelesof Adama woreda.The study units were randomly selected households from each cluster in five selected rural kebeles of Adama woreda.The study was carried out from February to August, 2016.

\subsection{Sampling Methods}

A total of 37 rural kebeles started community led total sanitation program, only 10 kebeles were graduated open defecation free in the study areas. Of 10 kebeles, five were selected by simple random sampling (lottery method) and the sample size was distributed proportionally to each of them based on the number of households they have. The five rural kebeles were more clustered in to 'gares' and proportional allocations of sample were assigned to the respective 'gares'. The five kebeles have 68 'gares' with average 1,700 households which mean each 'gare' has 20 up to 30 on average 25 households. I was assigned 604 households sample size proportionally to five kebeles which reached almost 25 households per clusters and the left 8 households were distributed by simple random sampling system.

\subsection{Sample Size Determination}

The sample size was determined using a single population proportion formula.

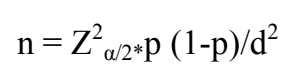

Where

a. $\mathrm{n}=$ Sample size of households.

b. $\mathrm{P}=$ Proportion of households ended open defecation free or achieved community led total sanitation. No previous similar study was carried out in the area. So, to get maximum sample size, $\mathrm{P}$ was taken as $50 \%(\mathrm{P}=0.5)$.

c. $\mathrm{d}=$ Degree of accuracy required (sampling error) is $5 \%$ that is, $\mathrm{d}=0.05$.

d. $Z=$ Standard score for $95 \%$ confidence level is 1.96 .

e. Additional $5 \%$ for non-response rates and design effect 1.5 were taken.

Substituting the above values (1), the calculated sample size for the study was 605 households.

\subsection{Inclusion and Exclusion Criteria}

Inclusion sampling criteria for this study was households living in the selected rural kebeles for greater than six months before the study begin and volunteer to participate in the study.Exclusion criteria for this study were individuals who reside in the selected kebeles for less than six months and who did not volunteer to participate in the study. The other exclusion sampling criteria were institutions such as offices, hotels, super markets and etc.

\subsection{Ethical Considerations}

Ethical approval and clearance was obtained from the
Institutional Review Board of Oromia Health Bureau after Adama General Hospital and Medical College wrote official letter. Formal letter of cooperation was written for Adama district Health Office. Consent of district health office and respective kebeles were obtained. Informed consent was obtained from each study subject. Any involvement in the study was carried out with the full consent of the person being interviewed. Finally after collection of the necessary data, identified problems during an evaluation process were discussed with health office so as to improve community led total sanitation achievement in the district.

\subsection{Data Collection Techniques}

Data were collected using structured, pretested and face to face interviewer-administered questionnaires through house to house. Questionnaires were translated in to local languages (Afan Oromo) based on the data collectors and study area of the communities understands. Pre-testing of the instrument was done in order to identify the clarity of questions. Five percent of the questionnaires (sample size) were carried out in a rural kebele of BosetWoreda which is near and similar to study area before the real data collection start. Discussion was held with the interviewers and households on the problems they encountered to collect data during the pretesting. Correction was incorporated in the final questionnaire. Training was given for seven health extension workers who were recruited as data collectors and two health professionals who were employed as the supervisor for one day before the pretest and for a day after the pretest. The training includes the objectives of the study, method of data collection, how to check completeness of questionnaire and the way approaching to the households.

\subsection{Data Processing and Analysis}

After the collected data was compiled, organized, coded, summarized, entered using computer software EPI version 7 and exported to SPSS version 20. The data analyzed using bivariate and multivariate logistic regression to determine the effect of various factors on the outcome variable. The degree of association between independent and dependent variables were assessed using adjusted odds ratio with $95 \%$ confidence interval and $p$-value $\leq 0.05$.

\section{Results}

This study was done in five open defecation free posted rural kebeles of Adama district of East Shoa zone, Oromia region. A total of 605 households, $594(98.3 \%)$ of a response rate of this $377(63.5 \%)$ females and $217(36.5 \%)$ males were included in this study. Among these 410 (69\%) were age greater than 50 years. Majority of respondents, 534 (89.9\%) Oromo by ethnicity, 430(72.4\%) orthodox in religious and $300(50.51 \%)$ were house wives in occupation. 
Table 1. Socio-demographic characteristics in rural kebeles of Adama district, East Shoa Zone, Oromia Region, Ethiopia, August, 2016. (n=594).

\begin{tabular}{|c|c|c|c|}
\hline Variables & & Frequency & Percent \\
\hline \multirow{5}{*}{ Age } & $20-29$ & 81 & 13.64 \\
\hline & 30-39 & 224 & 37.71 \\
\hline & $40-49$ & 131 & 22.05 \\
\hline & $50-59$ & 75 & 12.63 \\
\hline & $\geq 60$ & 83 & 13.97 \\
\hline \multirow{3}{*}{ Sex } & Male & 217 & 36.5 \\
\hline & Female & 377 & 63.5 \\
\hline & Oromo & 534 & 89.9 \\
\hline \multirow[t]{2}{*}{ Ethnicity } & Amhara & 45 & 7.6 \\
\hline & Others ${ }^{\mathrm{a}}$ & 15 & 2.5 \\
\hline \multirow{4}{*}{ Religion } & Muslim & 38 & 6.4 \\
\hline & Orthodox & 430 & 72.4 \\
\hline & Protestant & 98 & 16.5 \\
\hline & Other ${ }^{b}$ & 28 & 4.7 \\
\hline \multirow{6}{*}{ Educational } & Illiterate & 188 & 31.6 \\
\hline & Read and write & 116 & 19.5 \\
\hline & Grade 1-4 & 137 & 23.1 \\
\hline & Grade 5-8 & 126 & 21.2 \\
\hline & Grade $9-12$ & 24 & 4 \\
\hline & Certificate/diploma & 3 & 0.5 \\
\hline \multirow{5}{*}{ Occupation } & House wife & 300 & 50.51 \\
\hline & Merchant & 9 & 1.52 \\
\hline & Employee & 2 & 0.34 \\
\hline & Daily laborer & 37 & 6.22 \\
\hline & Other ${ }^{c}$ & 246 & 41.41 \\
\hline \multirow{2}{*}{ Monthly income } & $<150$ birr & 399 & 67.2 \\
\hline & $\geq 150$ birr & 195 & 32.8 \\
\hline \multirow{2}{*}{ Family size } & $\leq 5$ & 399 & 67.17 \\
\hline & $>5$ & 195 & 32.83 \\
\hline
\end{tabular}

Note: (Gurage, silte and Woliyita $\left.{ }^{\mathrm{a}}\right)$, (wakefata and Kalicha $\left.{ }^{\mathrm{b}}\right)$, farmers ${ }^{\mathrm{c}}$

Among total study, only $51(8.6 \%)$ of the respondents answered that community led total sanitation was achieved.

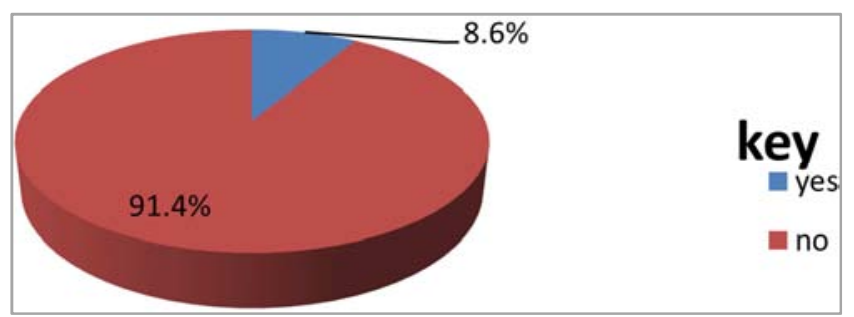

Figure 1. Community led total sanitation achievements among interviewed households in ruralkebeles of Adama district ( $n=594)$, July, 2016.

Out of the $370(62.3 \%)$ households who have latrines, only $69(11.6 \%)$ improved latrines (pit latrine with slabs) which were used only by family members. Majority of $(92.7 \%)$ the respondents have their own latrines and 47 (68.1\%) use improved latrines.

Table 2. Percent distribution of household's utilizing improved latrine in rural kebeles of Adama district, East Shoa Zone, Oromia Region, Ethiopia, August, 2016. $(n=594)$.

\begin{tabular}{lll}
\hline Variables & Frequency & Percent \\
\hline Availability of latrine & & \\
Pit with slab (improved) & 69 & 11.6 \\
Pit without slab (Non improved) & 301 & 50.7 \\
$\begin{array}{l}\text { The owner of the latrine } \\
\text { Private }\end{array}$ & 343 & 92.7 \\
\hline
\end{tabular}

\begin{tabular}{lll}
\hline Variables & Frequency & Percent \\
\hline Communal & 27 & 7.3 \\
The reason not having latrine & & \\
Lack of space & 21 & 9.4 \\
High cost & 105 & 46.9 \\
Create bad smell & 31 & 13.8 \\
Attracts flies, rats and insects & 64 & 28.6 \\
Not needed by households & 3 & 1.3 \\
Latrine facility household usually use & & \\
Non improved latrine (pit latrine without slab & 229 & 76.1 \\
Improved latrine (Open pit with slab) & 47 & 68.1 \\
The main benefit of latrine & & \\
For privacy & 75 & 20.3 \\
For satisfaction & 33 & 8.9 \\
For prevention of diseases & 203 & 54.9 \\
For dignity & 49 & 13.2 \\
For clean environment from human feaces & 10 & 2.7 \\
\hline
\end{tabular}

According to this finding result, 216 (58.38\%) have hand washing facilities. Also from those households have hand washing facilities, $178(30 \%)$ washed their hands after using the toilet with soap and water. Among washed their hands after visited latrines, majority $487(82 \%)$ washed their hands sometimes whereas the rest always.

Table3. Percent distribution of households' hand washing practice after utilization of latrine among study respondents in rural kebeles of Adama district, East Shoa Zone, Oromia Region, Ethiopia, August, 2016. (N=594).

\begin{tabular}{lll}
\hline Variables & Frequency & Percent \\
\hline Hand washing practice & & \\
After using latrine & 178 & 30 \\
Before preparing food & 263 & 44.3 \\
Before preparing food & 559 & 94.1 \\
After work and shaking people & 302 & 50.8 \\
How often wash your hand & & \\
Always & 102 & 17.2 \\
Sometimes & 492 & 82.8 \\
\hline
\end{tabular}

Of the respondents $568(95.6 \%), 18(3 \%)$ and $8(1.2 \%)$ were used public stands, protected dug wells and unprotected sources (rivers, ponds, damps and unprotected wells) respectively. $584(98.3 \%)$, of the households fetched water outside their housing compounds, of these 549 (92.4\%) travel greater than or equal to 30 minutes to fetch water (please see table 4 below).

Table 4. Households access to improved water supply among interviewed households in ruralkebeles of Adama district (n=594), August, 2016.

\begin{tabular}{lll}
\hline Variables & Frequency & Percent \\
\hline $\begin{array}{l}\text { Source of drinking water } \\
\text { Public stands }\end{array}$ & 568 & 95.6 \\
Dug well protected & 18 & 3 \\
Unprotected dug well & 8 & 1.2 \\
Households fetched water & & \\
Outside their housing compounds & 584 & 98.3 \\
Inside their housing compounds & 10 & 1.7 \\
Taking time to fetch water (go and come back) & & \\
$<30$ minute & 35 & 7.6 \\
$\geq 30$ minutes & 549 & 92.4 \\
Problems do you face in relation to water & & \\
Scarcity/absences & 490 & 62.5 \\
Water borne diseases & 215 & 36.2 \\
Expensive payment & 90 & 15.2 \\
Distance from their home to water sources & 372 & 62.6 \\
\hline
\end{tabular}


Of the respondents replied on community led total sanitation achieved $51(8.6 \%)$, only $27(52.9 \%)$ revealed that they recognized step of community led total sanitation (triggering).

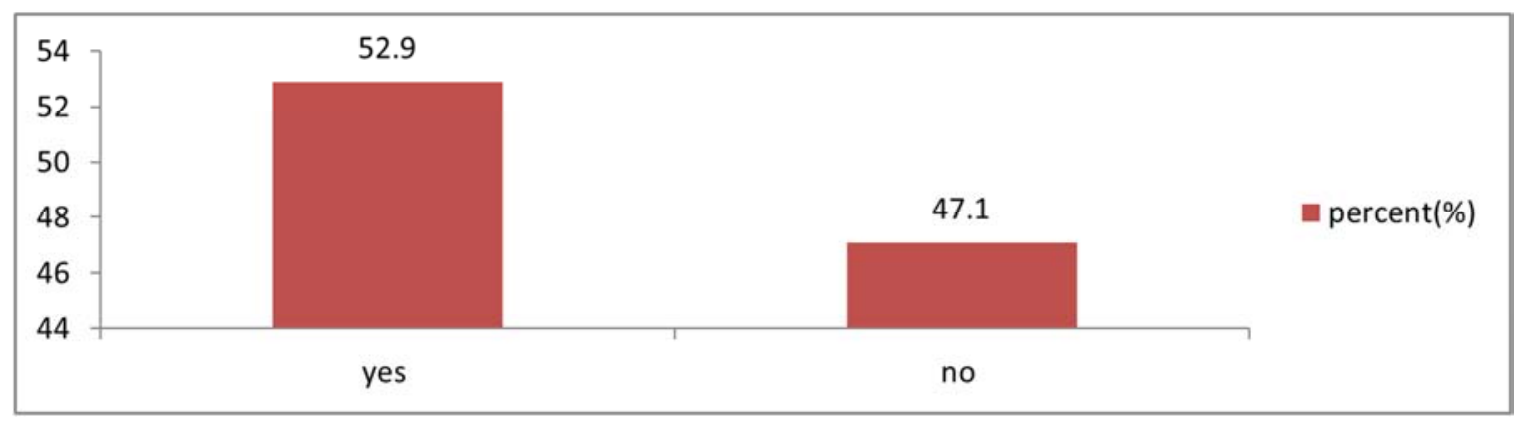

Figure 2. These Knew and participated on triggering step among interviewed households in ruralkebeles of Adama district (n=594), August, 2016.

Table 5 below indicates factors like sex of the respondents, monthly income of the respondents, not open dumped in yard and not knew steps of community led total sanitation were associated with community led total sanitation achievement after Bivariate analysis was carried out to examine the associated factors for community led total sanitation achievements at the household level. Many variables were explored to test association of community led total sanitation achievements like sex of the respondents, monthly incomes of respondents and households attended in steps of community led total sanitation achievements like waste disposal systems (open dump in yard), triggeringandcommunity conversationstatistically have significant association.

Adjustment of variables using multivariate logistic regression was carried out to predict community led total sanitation achievements variables; sex of the respondents (AOR $=4.72,95 \% \mathrm{CI}=1.09-20.48$, monthly income of the respondents $(\mathrm{AOR}=2.00,95 \% \mathrm{CI}=1.09-3.69)$, not open dumped in yard $(\mathrm{AOR}=2.03,95 \% \mathrm{CI}=1.11-3.72)$ and not knew triggering step $(\mathrm{AOR}=3.54,95 \% \mathrm{CI}=1.41-8.89)$ were significantly associated with community led total sanitation achievements.

Table 5. Analysis on associated factors with community led total sanitation achievements among the respondents in rural kebeles of Adama district, August, 2016. $(n=594)$.

\begin{tabular}{|c|c|c|c|c|}
\hline \multirow{2}{*}{ Variables } & \multicolumn{2}{|c|}{ CLTS achievement } & \multirow{2}{*}{$\operatorname{COR}(95 \% \mathrm{CI})$} & \multirow{2}{*}{$\operatorname{AOR}(95 \% \mathrm{CI})$} \\
\hline & Yes & No & & \\
\hline \multicolumn{5}{|l|}{ Sex of the respondents } \\
\hline Male & $18(8.3 \%)$ & $199(91.7 \%)$ & 1 & 1 \\
\hline Female & $33(8.8 \%)$ & $344(91.2 \%)$ & $2.28(1.28-4.07)$ & $4.72(1.09-20.48)^{*}$ \\
\hline \multicolumn{5}{|c|}{ Households in triggering step of community led total sanitation achievement } \\
\hline Knew and participated & $27(33.3 \%)$ & $54(66.7 \%)$ & 1 & 1 \\
\hline Not Knew and participated & $24(4.7 \%)$ & $489(95.3 \%)$ & $2.11(1.06-4.23)$ & $3.54(1.41-8.89)^{* *}$ \\
\hline Yes & $22(6.3 \%)$ & $326(93.7 \%)$ & 1 & 1 \\
\hline No & $29(11.8)$ & $217(88.2 \%)$ & $2.37(1.31-4.26$ & $2.03(1.11-3.72)^{* *}$ \\
\hline \multicolumn{5}{|l|}{ Community conversation } \\
\hline Yes & $10(3.5 \%)$ & $278(96.5 \%)$ & 1 & 1 \\
\hline No & $41(13.4 \%)$ & $265(86.6 \%)$ & $1.58(0.88-2.83)$ & $0.28(0.09-1.66)$ \\
\hline \multicolumn{5}{|c|}{ Monthly income in birr of households head } \\
\hline$\geq 150$ & $15(7.7 \%)$ & $185(92.3 \%)$ & 1 & 1 \\
\hline
\end{tabular}

Note: Significant at $(* \mathrm{P}<.05 ; * * \mathrm{P}<0.01 ; * * * \mathrm{P}<0.001)$, AOR: Adjusted Odds Ratio, COR: Crude Odds Ratio, CI= confidence interval.

\section{Discussion}

This study revealed that majority $(62.3 \%)$ of the households had access to latrine. This is slightly greater by $3.9 \%$ than the study conducted (58.4\%) in BahirdarZuria district of North Ethiopia in 2013 [13] whereas less by $31.7 \%$ and $6.7 \%$ than the study shown in SNNP region of Ethiopia at Mirab Abaya (94\%) and Alaba (69\%) respectively [11]. The study conducted at North Ethiopia of Denbia district displayed $(86.8 \%)$ greater [14] by $24.5 \%$ than this recent finding. The current study result $(11.6 \%)$ on improved latrines (pit latrine with slabs) coverage is higher via $9.6 \%$ than the Ethiopia mini demographic health survey conducted $(2 \%)$ in rural areas in 2014 . These differences may be due to available locally materials for latrine construction, continuous training, support and follow up of health extension professionals.

Joint monitoring program for water supply and sanitation by WHO and UNICEF in 2015 identified protected well and public tap/stand pipes are improved water sources among others [2]. Accordingly this study, 3\% used protected dug wells which is less than by $13 \%$ revealed in study conducted (16\% used protected dug well) on microbiological evaluation of the efficacy of soapy water to clean hands in 2014 [18]. This may be due to geographic, lack of awareness toward use 
of improved water sources and lack of access improved water supply sources.

Regarding time to fetch water from sources, majority $(92.4 \%)$ of households answered that it takes greater than 30 minutes. In rural areas studied done by Ethiopia Demographic and Health Survey 2011, shows 62\% [19] and according to WHO guideline recommends less than 30 minutes [1]. This finding shows greater than by $30.4 \%$ study result done by Ethiopia Demographic and Health Survey 2011. The reason could be due to scarcity or inadequate water $(82.5 \%)$ and distance between water source and their houses $62.6 \%$ as the proportion of this research shows, others may be quality of water accessed to them and scatter of houses among each other's.

With reference to hand washing facilities, more than half of the respondents $(58.38 \%)$ have hand washing facilities according to this finding. This finding higher almost by 3.4 , 2.2 and 9.4 times than study conducted in an eleven country study and found in structured observations that $17 \%$ of care givers washed hands with soap after defecation [22], Denbia (26.9\%) [14] and Baher Dar Zuria (6.2\%) [13] Districts respectively. The study showed only $30 \%$ of the households with latrines washed their hands with soap/ash and water after defecation. It is higher via $21.8 \%$ and $13 \%$ than the study conducted in kersa district (8.2\%) [25] and eleven countries $(17 \%)$ respectively [22]. This difference may be due to the fact that recently there has been high mobilization of the community on hygiene and sanitation which increases hand washing facility coverage of the study area.

This study shown that, among 594 interviewed households on community led total sanitation achievement, majority (91.4\%) of the respondents replied it was not achieved. This finding result showed that 1.4 higher than the proportion of the population practicing open defecation which was documented by Joint Monitoring Program of WHO and UNICEF at Geneva, Switzerland (90\%) (2015) [2] and 32.4 times higher than $59 \%$ of the one WASH Plus program by the Government of Ethiopia and UNICEF in rural kebeles Abomsa town (2014) [17]. Of this this finding, 513 (86.4\%) shown that they did not know its step (triggering) of implementation. In this resulted, most of population practicing open defecation. This may be due to community led total sanitation steps was not well implemented, lack of community mobilization, no bylaws, less community conversation on community led total sanitation, low support and follow from health extension workers.

Based on the result of multivariate analysis from sociodemographic respondents have less income $(<150$ birr) can decrease community led total sanitation achievement by two times than households with monthly income greater or equal birr 150 per month. This may be due households got greater or equal birr 150 per month have ability to achieve community led total sanitation than these lower incomes. Female's households head can increase community led total sanitation achievement by 4.72 times than males according this finding. This might be due to females have a higher responsibility in their households than females. Regarding to households not know and attended on triggering 3.54 times decreasing than these know and attended. It may be due to these got adequate awareness on step (triggering) of community led total sanitation can increase achievement. Households not disposed wastes in yard increase by 2.03 times than these disposal wastes in yard the achievement of community led total sanitation. This may due to the dispose in open sites.

\section{Limitation of the Study}

The households that were included in the study were from only one rural district (Adama) and other rural districts in oromia were not incorporated in this study. Research results therefore are limited to this particular rural district and cannot be generalized to other districts in Oromia.

\section{Conclusion and Recommendations}

This study revealed that most of the household's the latrine coverage was low $(62.3 \%)$ of this only $11.6 \%$ household have and used improved latrines whereas the study have only $30 \%$ hand washing practices after utilizing latrines. Regarding to access to water supply in the study area, majority $(92.4 \%)$ of the households were moved more than or equal to 30 minutes for fetching water from sources which was greater than the WHO guidelines.

Community led total sanitation achievement in the five selected open defecation free kebeles was $8.6 \%$. This result showed indirectly that $91.4 \%$ of community led total sanitation not achieved in the study areas which was higher. Therefore, based on this finding, I concluded that generally community led total sanitation achievement in the study areas were very lower than the Adama woreda health office annually updated (2105) report (100\% verified end of open defecation), World Health Organization and United Nation Children Funds updated report in 2015. Regarding latrine utilization, hand washing practice after utilizing latrines and access to improved water supply were very lower than what was being reported by Adama woreda health office 2015) which was $100 \%$ of latrine coverage as well as utilizations, hand washing practices after visiting latrines and access of improved water supply less than 30 minutes. Therefore, this study recommends the following actions in order to alleviate the problems:

a. Additional improved water supply sources should be provided to supply adequate and continues water to the community.

b. Increasing community based health education on hand washing practices after defecation is essential by strengthening health extension workers.

c. Increasing awareness and practices of safe solid waste disposal methods by the households and preparation of solid waste disposal site by the town municipal.

d. Re implement community led total sanitation following its steps and continues follow-up, support, mobilize and supervision by all stakeholders.

e. Further research is needed 


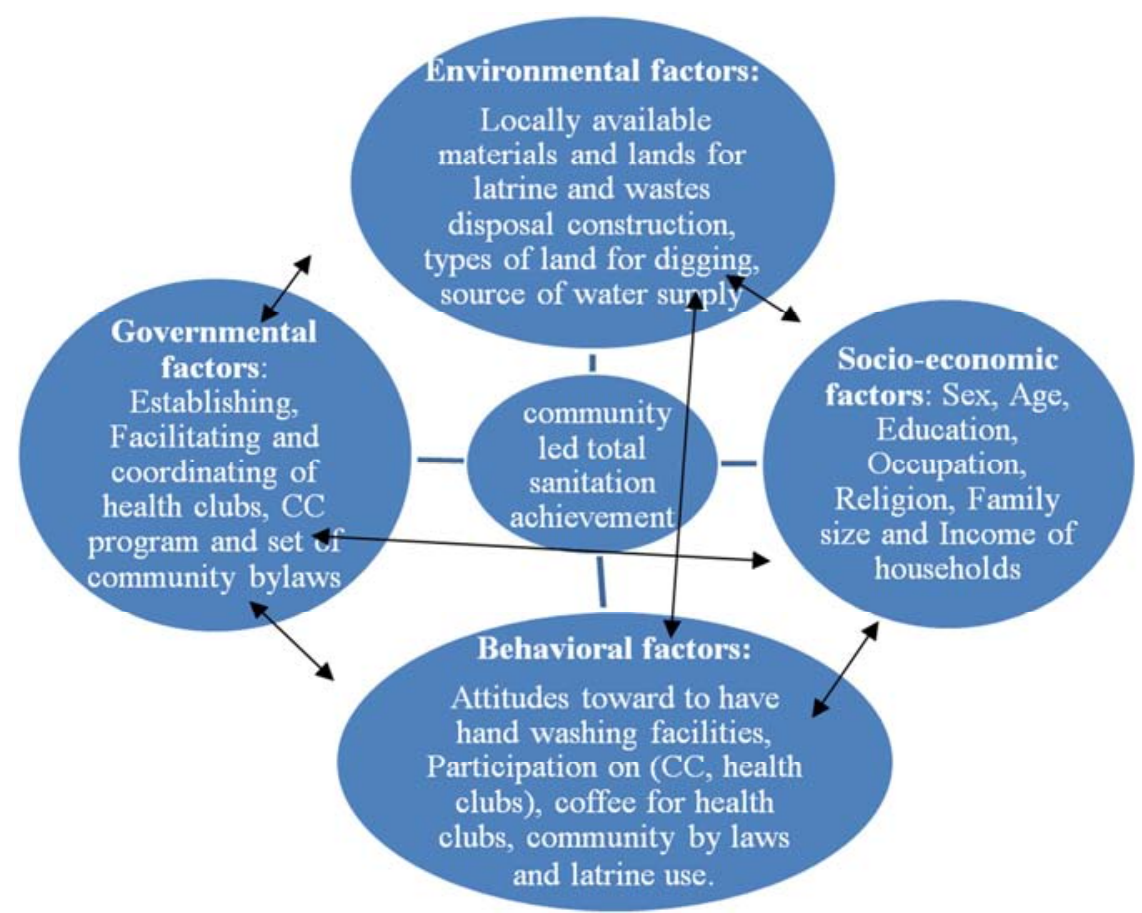

Figure 3. Conceptual frame work of community led total sanitation achievement and its associated factors in five open defecation free rural kebeles of Adama district, East Shoa Zone, Oromia Region, Ethiopia, August, 2016. (n=594).

\section{References}

[1] UNICEF and WHO: Progress on Drinking Water and Sanitation, 2012 updated.

[2] WHO/UNICEF Joint Monitoring Programme: Progress on Drinking Water and Sanitation report. Geneva, Switzerland: 2015.

[3] Esrey et al, Effects of improved water supply, sanitation and hygiene. Bulletin of the Royal Society of Tropical Medicine and Hygiene, 2011, Vol. 77, No 4, pp. 5151-521.

[4] Barnard S, Routray P, Majorin F, et al. Impact of Indian Total Sanitation Campaign on Latrine Coverage and Use. 2013.

[5] Jee HR, A CA, Bhupendra B, et al. Household sanitation and personal hygiene practices are associated with child stunting in rural India: a cross-sectional analysis of surveys. 2015:1.

[6] Kamal K, Chambers R. Handbook on Community-led Total Sanitation

http://www.communityledtotalsanitation.org/resource/handboo k-community-led-total-sanitation IDS and Plan International.2008.

[7] Sameer S, Amsalu N. Community led total sanitation (CLTS): Addressing the challenges of scale and sustainability in rural Africa 2008:2-6.

[8] Deise G, Seung-Sup K, Jay G. Exploring changes in open defecation prevalence in sub-Saharan Africa based on national level indices 4. BMC Public Health. 30 May, 2013.

[9] WHO. Rural water and sanitation assessing impacts. June 2015.

[10] Ethiopia Mini Demographic and Health Survey (EMDHS).
2014.

[11] Behailu S, Redaie G, Mamo D, et al. Promoting sanitation and hygiene to rural households in the Southern Nations, Nationalities and Peoples Region, Ethiopia. May, 2010(15).

[12] Supporting the Health Extension Program. Addis Ababa, Ethiopia Pathfinder International, January, 2012.

[13] Worku A, Semahegn M. latrine coverage and associated factors among rural communities in the District of Bahir Dar Zuria, Ethiopia. 2013:4.

[14] Yimam T, Kassahun A, Daniel H. Latrine utilization and associated factors among people living in rural areas of Denbia district, Northwest Ethiopia. 2013:10.

[15] Yohannes T, Workicho A, Asefa H. Availability of Improved Sanitation Facilities and Associated Factors among Rural Communities in LemoWoreda, Hadiya Zone, Southern Ethiopia. 2014.

[16] Abebe B, Tamene H, FarisKebede, et al. Current state and trends of access to sanitation in Ethiopia. 2015.

[17] The One WASH Plus programme is jointly implemented by the Government of Ethiopia and UNICEF in Abomsa, Oromia. October 2014.

[18] Amin N, Pickering A, Ram P, Unicomb L, et a Microbiological evaluation of the efficacy of soapy water to clean hands: a randomized, non-inferiority field trial. Am J Trop Med Hyg. PubMed. 2014; 91(2):415-23.

[19] Ethiopia Demographic and Health Survey 2011.

[20] Mohammed A, Zungu L, Hoque M. Access to Safe Drinking Water and Availability of Environmental Sanitation Facilities among Dukem Town Households in Ethiopia. Hum Ecol. 2013; 41(2):131-8. 
[21] Solomon B, Srilaxmi S. An Assessment on the Status of Water Supply and Sanitation in Ethiopia: A Case of Ambo Town. Journal of Sustainable Development in Africa. 2011; 13(1).

[22] Curtis V, Danquah L, Aunger R. Planned, motivated and habitual hygiene behaviour: an eleven country review. Health Educ Res.PubMed. 2009; 24(4):655-73.

[23] Martin M, Canada D, Salinero F, etal. Effectiveness of a training programme to improve hand hygiene compliance in primary healthcare. BMC Public Health. 2009; 9:1471-2458.

[24] Biran A, Schmidt W, Zeleke L, Emukule H, et al. Hygiene and sanitation practices amongst residents of three long-term refugee camps in Thailand, Ethiopia and Kenya. Trop Med Int Health. PubMed. 2012; 17(9):1133-41.

[25] MengistieBB. Community based assessment on household management of waste and hygiene practices in KersaWoreda, Eastern Ethiopia. 2010; Ethiop J Heal Dev. (2):103-9.

[26] Lyla M, Petra B. Community-led total sanitation (CLTS) across the seas: Experiences from Africa with a special emphasis onEthiopia. october 2009(12).

[27] OfficeAdama Woreda Health. Annual report.unpublished document from the District Health Office ofAdama. 2015/2016. 2015 . 